\title{
Is a novel human retrovirus associated with prostate cancer and chronic fatigue syndrome?
}

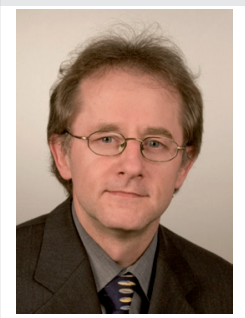

“...reports of a new, probably oncogenic

human retrovirus have recently been published.

Unexpectedly, the novel family member

was clearly shown to be a

classical gammaretrovirus..."

Nobert Bannert

Centre for Biological Safety 4, Robert Koch Institute, Nordufer 20, 13353 Berlin, Germany

- Tel. : +49 3045472549 m. Fax: +49 3045472334 m BannertN@rki.de

Almost in time for the centennial of Ellerman and

Bang's seminal discovery that avian leukemia can be induced by a transmissible retrovirus [1], reports of a new, probably oncogenic human retrovirus have recently been published [2]. Unexpectedly, the novel family member was clearly shown to be a classical gammaretrovirus, a genus known to infect rodents, cats, gibbons, koalas and some other animals, but not humans. However, human genomes tell a story of primeval gammaretroviral infections, with some gammaretrovirus-type elements having integrated in the germ line of our ancestors millions of years ago, the remnants of which we still carry as part of the human endogenous retrovirus legacy that makes up an astonishing $8 \%$ of our chromosomal DNA [3].

Based on the high sequence similarity with xenotropic endogenous murine leukemia viruses (MLVs) present in the genomes of mice, the newly discovered gammaretrovirus has been named xenotropic MLV-related virus (XMRV). A xenotropic virus is no longer able to infect the cells of its natural host but can infect those of many other species, including humans. How could such a situation arise? Most likely, the selective pressure exerted by the virus led to the increased survival of those mice bearing mutated cellular receptors unable to mediate viral entry despite already having replication-competent endogenous viruses integrated into their chromosomes. Such mice can be regarded as 'resistant spreaders' of an infectious endogenous retrovirus carried as provirus in their genomes.

Replicating gammaretroviruses such as $\mathrm{XMRV}$ that do not encode oncogenes are known to cause cancers due to their tendency to integrate close to or into transcriptionally active sites. If a tumor suppressor gene or proto-oncogene is affected, such insertional mutagenesis can induce or promote neoplastic transformation. This was tragically and unintentionally demonstrated in humans by the development of leukemia in three children following gene therapy with an MLVbased vector. The leukemic cells from two of the children were found to carry the viral vector just upstream of the LMO2 oncogene [4]. However, alternative mechanisms for retroviral tumorigenesis and the implications for neurologic and autoimmune diseases are also being investigated, including the possible involvement of the viral envelope protein and a role for paracrine mechanisms and chronic inflammation.

Therefore, it was of considerable concern that in the initial report XMRV was found to be present in $40 \%$ of prostate cancer biopsies from US individuals that had a familial history of this disease and homozygosity for an $R N$ ase $L$ gene coding for a glutamine at position 462 (R462Q) [2]. This variant displays a threefold reduced catalytic activity compared with the regular enzyme carrying an arginine at this position. RNase $\mathrm{L}$ is an endoribonuclease of the innate antiviral response system activated by interferons. The QQ genotype is present in $11 \%$ of the population and has been shown to be associated with an up to twofold increased risk of developing prostate cancer. This disease, with almost 200,000 cases and more than 27,000 deaths each year in the USA alone, is one of the most common forms of malignancy known. Indeed, worldwide approximately $3 \%$ of males die of prostate cancer. The link between the interferon-sensitive XMRV and the R462Q RNase L variant was strong because evidence of the virus has only been found in $1.5 \%$ of prostate cancer patients with an RR genotype or heterozygosity at this locus [2]. Therefore it appeared quite plausible that the presumably

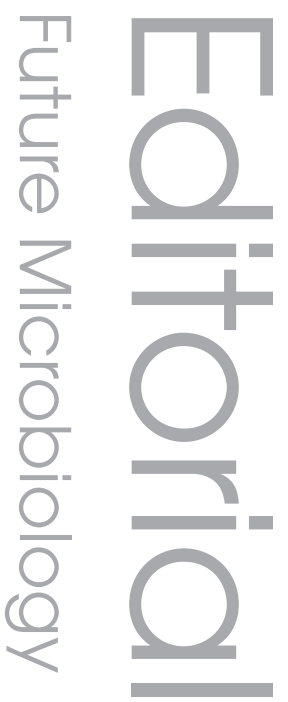

\section{future}


impaired branch of the antiviral innate immunity in individuals with QQ alleles would favor infection with XMRV.

However, this hypothesis was severely challenged by an independent study showing no link between XMRV and the RNase L genotype but a positive correlation with the malignant grade of the carcinoma [5]. Even more importantly, discordant results were also obtained regarding the type of cell infected in the prostate gland. Using immunocytochemistry and polyclonal sera, Schlaberg and coworkers revealed the presence of XMRV proteins in epithelial tumor cells but rarely in stromal cells. In the initial work by Urisman and colleagues, in situ hybridization and immunostaining failed to localize the new gammaretrovirus in the proliferating epithelial prostate tumor cells (the virus certainly relies on cell division for successful infection) but only in a few adjacent stromal cells [2]. Although this does not exclude an etiologic implication in cancer development per se (XMRV-infected stromal cells could, for instance, induce neoplasia of surrounding epithelia by paracrine mechanisms or provoke chronic inflammation eventually resulting in cell transformation), the presence of a retrovirus in the genome of the malignant cell is more compatible with the better-understood mechanisms of cell transformation by insertional mutagenesis. Interestingly, viral proteins or DNA were detected in $27 \%$ of the 334 prostate cancer cases described in the latter study [5] by immunocytochemistry or quantitative PCR and although this stimulated further the interest in XMRV, demonstration of a causal link between infection and prostate cancer remained elusive.

The considerable prevalence rates reported in these two independent studies from the USA were somewhat shaken by data from northern European cohorts. Remarkably, a complete lack of the virus in 589 prostate cancer tumors has been reported in one study [6] whereas only one out of 105 prostate tumor samples and one out of 70 healthy controls were XMRV-positive in another German study [7], despite both studies using very sensitive PCR assays for detection. Furthermore, in the larger study, 146 sera samples from prostate tumor patients tested negative for virus-specific antibodies using a newly developed ELISA [6].

Xenotropic murine leukemia virus-related virus once again came into the focus recently when a research team lead by Judy Mikovits from the Whittemore Peterson institute in Reno (NV, USA) detected XMRV in the blood of approximately two-thirds of chronic fatigue syndrome
(CFS) patients and almost $4 \%$ of healthy controls [8]. A number of patients were also shown to have mounted an XMRV-specific immune response. CFS is not a rare condition, with approximately $1 \%$ of the world's population suffering from the incapacitating fatigue that is often linked to chronic inflammation. The controversy in the medical community as to whether CFS is even a distinct disease and the lack of a firmly established cause contributes significantly to the desperation felt by many of those stricken by the syndrome. The possibility of a link to XMRV was therefore met with a great deal of excitement and sparked hope for the development of effective treatment or prevention strategies against CFS. Moreover, the high frequency of XMRV infection reported for normal individuals suggested a significant, but previously unrecognized threat, with 10 million people in the USA alone being infected by a gammaretrovirus of largely unknown pathogenic potential. Authorities responsible for the safety of blood products and for transplantations, having been alerted by the Science report [8], examined their records and concluded that there is no known association between CFS or prostate cancer and a history of transfusion. The US Department of Health and Human Services has nevertheless taken action and rapidly formed an interagency scientific working group on XMRV to investigate the potential threat for blood products posed by this virus.

Therefore, it is with some sense of relief that three recent publications from two research groups in the UK and one from The Netherlands reported a failure to detect XMRV sequences in 186 [9], 299 [10] and 32 [11] CFS patients using sensitive PCR methods. The British patients in the Erlwein et al. study [9] met the criteria for CSF set by the Centers for Disease Control that were also fulfilled by the cohort in the Science paper [8]. Therefore, similar to the situation with XMRV and prostate cancer, the link between the virus and CFS remains highly contentious and causality is far from having been proven.

It should be kept in mind that conflicting results, controversy and skepticism during the early stages of a new discovery are not uncommon. What are the likely reasons for the contradictory results with regard to a link between $\mathrm{XMRV}$ and human disease and can these results be reconciled? Conceivably, the lack of standardized PCR methodology and the use of antibodybased diagnostic methods with variable detection limits might play a role. However, the XMRV sequences published so far are virtually identical, making it extremely unlikely that the virus might 
be missed using different PCR set-ups as they are each shown to detect less than ten proviral genome equivalents. Furthermore, that fact that the composition of the patient cohorts was not precisely the same in each study (e.g., the ratio of familial versus sporadic cases in prostate cancer or the criteria for CFS diagnosis) might contribute to differences in overall frequencies it can hardly explain the 'null virus' results from some studies.

One possible reason to explain the dramatic differences in studies from the USA and Europe would be a wide-ranging regional difference in XMRV distribution. Based on the relative lack of genetic diversity in the available XMRV sequences, the cross-species transfer(s) of XMRV to humans probably occurred fairly recently and the virus can be assumed to replicate only poorly in its new host. Infections of susceptible individuals might therefore be far more frequent in those regions that overlap with the habitats of the elusive natural host that carries the ancestral provirus in its germ line. If so, one would expect clusters of disease in such high prevalence regions associated with an infectious agent. However, for prostate cancer at least, the incidence rates reported for Caucasian populations in the USA and northern Europe are comparable.

Although the recent studies cast some doubt on the high prevalence rates previously reported, there is evidence to suggest that XMRV is not simply a laboratory contaminant. So far, the virus has been found in patient samples from different regions in the USA [2,5], Germany [7]

\section{Bibliography}

1. Ellermann V, Bang O: Leukämie bei Hühnern. Zentralbl. Bakteriol. Parasitenkd. Infectionskr. Hyg. Abt. Orig. 46, 595-609 (1908).

2. Urisman A, Molinaro RJ, Fischer $\mathrm{N}$ et al: Identification of a novel Gammaretrovirus in prostate tumors of patients homozygous for R462Q RNASEL variant. PLoS Pathogens 2(3), E25 (2006).

3. Bannert N, Kurth R: Retroelements and the human genome: new perspectives on an old relation. Proc. Natl Acad. Sci. USA 101(Suppl. 2), 14572-14579 (2004).

4. Hacein-Bey-Abina S, Von Kalle C, Schmidt M et al:: LMO2-associated clonal $\mathrm{T}$ cell proliferation in two patients after gene therapy for SCID-X1. Science 302 (5644), 415-419 (2003).

5. Schlaberg R, Choe DJ, Brown KR, Thaker HM, Singh IR: XMRV is present in malignant prostatic epithelium and is associated with prostate cancer,

and Japan [12] applying a variety of diagnostic methods. Furthermore, the known XMRV sequences are not completely identical, but at least to some extent, polymorphic and antibodies to XMRV proteins have been detected in some individuals. Finally, the human prostate tumorderived cell line $22 \mathrm{Rv} 1$ is known to be heavily infected with XMRV [13]. However, these points do not invalidate the understandable suspicion that XMRV and its association with human disease will eventually be added to the long list of related cases that evaporated under the scrutiny of subsequent investigation [14]. Although a great deal of excellent research on XMRV has been published recently, a plethora of urgent questions regarding transmission, origin, cell tropism animal models as well as potential prevention and treatment aspects, await answers. However, more immediate is the need for further prevalence studies using standardized assays to resolve the controversies surrounding XMRV and its ramifications for human disease.

\section{Financial \& competing interests disclosure \\ The author has no relevant affiliations or financial involvement with any organization or entity with a financial interest in or financial conflict with the sub- ject matter or materials discussed in the manuscript. This includes employment, consultancies, honoraria, stock ownership or options, expert testimony, grants or patents received or pending, or royalties. \\ No writing assistance was utilized in the production of this manuscript.}

especially high-grade tumors. Proc. Natl Acad. Sci. USA 106(38), 16351-16356 (2009).

6. Hohn O, Krause H, Barbarotto P et al.: Lack of evidence for xenotropic murine leukemia virus-related virus (XMRV) in German prostate cancer patients. Retrovirology 16(6), 92 (2009).

7. Fischer N, Hellwinkel O, Schulz C et al.: Prevalence of human gammaretrovirus XMRV in sporadic prostate cancer. J. Clin. Virol. 43(3), 277-283 (2008).

8. Lombardi VC, Ruscetti FW, Das Gupta J et al:: Detection of an infectious retrovirus, XMRV, in blood cells of patients with chronic fatigue syndrome. Science 326(5952), 585-589 (2009).

9. Erlwein $\mathrm{O}$, Kaye $\mathrm{S}, \mathrm{McCl}$ re $\mathrm{MO}$ et al.: Failure to detect the novel retrovirus XMRV in chronic fatigue syndrome. PLoS One 5(1), E8519 (2010).

10. Groom HC, Boucherit VC, Makinson K et al: Absence of xenotropic murine leukemia virus-related virus in UK patients with chronic fatigue syndrome. Retrovirology 7(1), 10 (2010).
11. van Kuppeveld FJ, Jong AS, Lanke KH et al:: Prevalence of xenotropic murine leukemia virus-related virus in patients with chronic fatigue syndrome in The Netherlands: retrospective analysis of samples from an established cohort. BMJ 340, C1018 (2010).

12. Furuta RA, Miyazawa T, Sugiyama T, Kimura T, Hirayama F: The prevalence of xenotropic murine leukemia virus-related virus in healthy blood donors in Japan. Presented at: 2009 Meeting on Retroviruses. Cold Spring Harbor Laboratory, NY, USA, 18-23 May, 2009 (Abstract).

13. Knouf EC, Metzger MJ, Mitchell PS et al.: Multiple integrated copies and high-level production of the human retrovirus XMRV (xenotropic murine leukemia virus-related virus) from $22 \mathrm{Rv} 1$ prostate carcinoma cells. J. Virol. 83(14), 7353-7356 (2009).

14. Voisset C, Weiss RA, Griffiths DJ: Human RNA "rumor" viruses: the search for novel human retroviruses in chronic disease. Microbiol. Mol. Biol. Rev. 72(1), 157-196 (2008). 